\title{
An Improved Model Reduction Method on AIMs for N-S Equations Using Multilevel Finite Element Method and Hierarchical Basis
}

\author{
M. Nauman Aslam*, Jiazhong Zhang, Nannan Dang and \\ Riaz Ahmad \\ School of Energy and Power Engineering, Xi'an Jiaotong University, Xi'an 710049, \\ China
}

Received 13 September 2017; Accepted (in revised version) 3 April 2018

\begin{abstract}
A numerical method is proposed to approach the Approximate Inertial Manifolds (AIMs) in unsteady incompressible Navier-Stokes equations, using multilevel finite element method with hierarchical basis functions. Following AIMS, the unknown variables, velocity and pressure in the governing equations, are divided into two components, namely low modes and high modes. Then, the couplings between low modes and high modes, which are not accounted by standard Galerkin method, are considered by AIMs, to improve the accuracy of the numerical results. Further, the multilevel finite element method with hierarchical basis functions is introduced to approach low modes and high modes in an efficient way. As an example, the flow around airfoil NACA0012 at different angles of attack has been simulated by the method presented, and the comparisons show that there is a good agreement between the present method and experimental results. In particular, the proposed method takes less computing time than the traditional method. As a conclusion, the present method is efficient in numerical analysis of fluid dynamics, especially in computing time.
\end{abstract}

AMS subject classifications: 35Q40, 76M10, 80M10, 35R01.

Key words: Fluid dynamics, model reduction, inertial manifolds, multilevel finite element method, hierarchical basis functions, nonlinear dynamics.

\section{Introduction}

In computational analysis, mathematical models which exhibit complex physical behavior along high fidelity and strong nonlinearity has been the focus of many researchers recently. In engineering, nonlinear continuous dynamical systems describe the vast majority of phenomena, such as complex fluid flows, fluid structure interactions. For fluid flow problems, the motion of flow exhibits complex behavior and behind this complexity is the fact that the dynamics of the systems may be the product of multiple different interacting

${ }^{*}$ Corresponding author. Email address: naumanaslamgcu@gmail . com (M. N. Aslam) 
forces. It is known that some dynamic systems with continuum mechanics, are governed by a set of nonlinear partial differential equations, and some nonlinear behaviors, which can be captured by numerical solution, occur in such kind of systems. Despite the complexity of the flow topology, the entire behavior of most fluid flows is described by the so-called Navier-Stokes equations. Since in most cases, these equations do not provide the known analytical solutions, many numerical methods have been developed over the years to solve them [1-5]. For such systems, when applying discretization methods, the cost of computing time for the resulting equations is considerably expansive due to high number of degrees of freedom. Normally, finite element method is applied to approach the solution to such governing equations. Consequently, the resulting equations are mostly nonlinear dissipative evolution equation with a lot of degrees-of-freedom. In order to analyze the dynamics of the equations, the system is changed into phase-space. However, in finite dimensional phase space with higher dimension, many difficulties appear from analyzing the nonlinear dynamics both qualitatively and quantitatively. Indeed, the disadvantage of the above mentioned numerical methods is that they require considerable computing time with great difficulties, due to large number of degrees-of-freedom, and the long term behaviors of the systems have great influences from the numerical computational errors [67]. For such problem, there are some numerical methods. For example, the systems with local nonlinearity have been analyzed by IRS and balanced realization methods by Friswell et al. [8]. In structural dynamics with nonlinearities, there are some reduction methods mostly having numerical algorithms based on component synthesis techniques which can be efficiently used for linear dynamic systems and the solution to these problems are obtained through many numerical experiments and computational analysis [9-10]. While Slaats et al. [11] introduced a reduction method based on three modes for nonlinear dynamical systems using finite element discretization. Therefore, model reduction for high dimensional or infinite dimensional fluid dynamic systems are required to overcome such difficulties.

On the other hand, in the study of long term behaviors of dissipative nonlinear evolution systems, one encounters with the global attractors, which is compact, invariant set with finite fractal dimensions attracting all the orbits of the systems uniformly, such attractors have complicated and dynamic structures [12]. A theoretical approach was shown that there is a approximate inertial manifold for the long term behavior of some dissipative partial differential equations in Titi [13]. Consequently, it has been proved that infinitedimensional dissipative systems can be reduced to finite dimensional systems by reduction technique. Thus, a number of methods have been applied to construct a finite system exhibiting asymptotic dynamic behavior in the original dynamic system [14-15]. More, an important feature of nonlinear dynamics is given related to model reduction, which explains the asymptotic properties based on spectral theory and decomposition process of the dynamical systems [16]. Therefore, developing a feasible model reduction method is very urgent as continuum dynamic systems are studied numerically.

For decades, the concept of inertial manifold is an important development in the study of systems with complicated attractor, since it reduces an infinite dimensional problem into a finite dimensional one without introducing errors [17]. Infinite dimensional dynamics 
sytems are converted into finite dimensional ones by applying the theory of inertial manifolds. It also reduces the computing time and space keeping the topology of the systems intact. The presence of inertial manifold has been verified and shown reducing the degree of freedom involving in the dynamic systems. This provides a strong mathematical basis for model reduction in fluid dynamical systems [18]. An inertial manifold is defined to be a finite dimensional Lipschitz manifold, which is invariant for a semi-group defined on a phase space and attracts all the orbits exponentially and uniformly for the given initial conditions on the bounded set. In fact, the study of integral manifolds and center manifolds lead to methods for the construction of IMs theoretically. Since then, some model or dimension reduction method are proposed. Among them, the approach to model reduction by the theory of Inertial Manifolds (IMs) is more powerful and has been carried out for different periods of time [19-20].

Theoretically, the presence of IMs usually sustains by very definitive spectral gap conditions. Therefore, practically the notion of Approximate Inertial Manifolds (AIMs) was introduced by Titi [13] and Foias et al. [21]. Haller and Sten [22] developed a model reduction method for the nonlinear mechanical systems by establishing certain conditions which can reduce the higher degrees of freedom to lower degrees of freedom. For this decomposition, they analyzed that there exists an exponential convergence defined on the slow manifold for the reduced model. For interaction between low and high modes, Schmidtmann [23] proposed a method using Approximate Inertial Manifolds by approximating the solution of MHD equation examining the behaviour of incompressible fluids. A Nonlinear Galerkin Method for coupling between lower and higher modes using proper orthogonal decomposition approached by Approximate Inertial Manifold was used by Kang et al. [24]. Laing et al. [25] applied AIMs for post processing calculation to lift the modes of the solution. On the other hand, some studies have been carried out for time-dependent second order autonomous dynamical systems which are nonlinear dissipative systems in nature by applying IMs and AIMs.

Further, some results have obtained by using AIMs approach for partial differential equations of second order depending upon time with delay [26]. So reduction of timedependent second order autonomous dynamical systems, which are nonlinear dissipative systems with many degrees-of-freedom, has been developed for infinite dynamical systems together with mode synthesis analysis, which are used mostly in engineering and has induced effects of model reduction on long term behaviors of dynamical systems. Also, the concept of inertial manifold with time-delay along with traditional Galerkin method exhibiting the dynamic behavior of nonlinear phenomena such as buckling of shallow arches under the load impact is introduced using AIMs [27].

Recently, AIMs approach was used for reduction of model for Navier-Stokes Equation using multilevel finite element method, and some numerical examples are given to verify the method by Zhang et al. [18]. For such problem, the numerical approach to the AIMs is the main point. In finite element method, the hierarchical element or basis can describe the addition of further nodal elements and has no effect on the preceding ones in a major way. Particularly, hierarchical representation is the series for the approximation of a function. The terms which are added in the series does not affect the previous ones. To attain a hier- 
archical shape function, every component is required for optimal shape function, variables, physical description and discretization used must be organized hierarchically, this concept has been impressively demonstrated in Whiting and Jansen [28]. Application of mesh refinement through adaptive finite element approximation has been applied, giving uniform refined results in a hierarchy of approximation spaces [29]. Derivation and analysis for solving boundary value problems using hierarchical basis multigrid method on rectangular regions is used in Bank et al. [30]. Following that, the multilevel finite element method with hierarchical basis can be available to approach the AIMs, and that is the main idea in this paper.

In this study, numerical method (Euler Scheme) is used to approach the unknown terms involved in the system of governing equations. The pressure-correction technique is applied numerically for the time derivative. The hierarchical basis is applied to the improvement in approaching AIMs, showing a broad set of hierarchical modes, which are decomposed into two subspaces, a finite dimensional space spanned by low modes and high modes. The flow dynamics is initially carried out by low modes rapidly converging onto AIMs characterized by high modes. The hierarchical basis functions are based on the construction for the specified variable $k$-order nodal meshes. Then, the finite element mesh is defined based on topological hierarchy of mesh entities. The decomposition of the solution space spanned by IMs is based on refinement of mesh domain by hierarchical finite element basis. Finally, to investigate the numerical method, dynamics of flow around an airfoil is carried out to show the accuracy and reduced computing time in comparison with the experimental results and traditional commercial software ANSYS(Fluent), showing a close agreement setting a benchmark for the problem.

\section{Problem formulation}

\subsection{Governing equations}

The Incompressible Navier-Stokes Equations with elementary variables are

$$
\begin{aligned}
& \frac{\partial u_{i}}{\partial t}+u_{j} \frac{\partial u_{i}}{\partial x_{j}}=-\frac{1}{\rho} \frac{\partial p}{\partial x_{i}}+v \frac{\partial^{2} u_{i}}{\partial x_{j} \partial x_{j}}, \\
& \frac{\partial u_{j}}{\partial x_{j}}=0
\end{aligned}
$$

where $u$ is the flow velocity, $p$ is the pressure, $v$ is the kinemetic viscosity, $\rho$ is the fluid density. The characteristics of flow depends on the non-dimensional parameters. The initial conditions $u\left(x_{i}, 0\right)=u_{0}\left(x_{i}\right)$.

\subsection{The boundary conditions}

We introduce the following system defining the set of boundary conditions $\Gamma=\Gamma_{1} \cup \Gamma_{2}$, where $\Gamma_{1}$ is boundary condition for velocity, $\Gamma_{2}$ boundary condition for pressure and on 
boundary $\Gamma_{1}$ and $u_{i}=\tilde{u}_{i}, i=1,2$ on boundary $\Gamma_{2}$. Also $p_{i j} n_{j}=\tilde{p}_{i}(i, j=1,2), n_{j}$ is

outward unit normal vector component and tensor $p_{i j}=-\frac{p}{\rho} \delta_{i j}+v\left(\frac{\partial u_{i}}{\partial x_{j}}+\frac{\partial u_{j}}{\partial x_{i}}\right)$.

\subsection{Existence of inertial manifolds}

A brief introduction to Inertial Manifolds and its existence is given in the following as we study the dynamical system generated by the evolution equation (2.2a). To approach inertial manifold, spectral method is normally used, and the governing equations are projected onto the space spanned by eigenfunctions of positive definite operator in terms of two components, namely small and large components.

Let $u$ be the solution to governing equation in an approximate Hilbert space. For model reduction, a mapping from higher modes to lower modes in the system is constructed.

$$
\begin{aligned}
& \frac{d u}{d t}+A u+E(u)=f, \quad t>0, \\
& u(0)=u_{0} .
\end{aligned}
$$

In Eq. (2.2a), $A$ is the space of self-adjoint linear operator $H, E(u)=E(u, u)$ corresponds to linear and low-order, $E(u, u)$ is also termed as bilinear operator. The Navier-Stokes Equations subject to boundary conditions can be written as abstract dissipative evolution equation given by (2.2a), where A is positive definite operator. As spectral method is used to approach Inertial Manifolds, the components involved for the projection are approached by eigenfunctions of the definite operator of the governing equations. Now if $\omega_{j},(j=$ $1,2, \cdots)$, denotes the eigenfunctions of the operator for the governing equations, gives $A \omega_{j}=\lambda \omega_{j}$, which leads to the construction of Inertial Manifolds in terms of components $\mathrm{P}$ and $\mathrm{Q}$. Since the existence of an IMs for Navier-Stokes Equations has not been proven, therefore it is obliged to use manifolds which are close to global attractors to approximate the permanent regime of dynamical systems. In this paper we provide AIMs approach to NSE, where Inertial Manifold for dissipative equations is obtained by modifying the Navier-Stokes Equations.

The subspace $P$ is spanned by the eigenfunctions or low modes and the subspace $Q$ is spanned by high modes. So, if inertial manifolds exists under the spectral gap condition, then we have a graph defined as $\phi: P \longrightarrow Q$.

Then $\phi: P_{m} H \longrightarrow Q_{m} H$ exists, where $P_{m}$ and $Q_{m}$ denote the orthogonal projections, i.e.,

$$
P_{m}: H \longrightarrow \operatorname{span}\left\{\omega_{1}, \omega_{2}, \cdots, \omega_{m}\right\}, \quad Q_{m}=I-P_{m} .
$$

So the projection is obtained as

$$
u(t)=p_{m}(t)+q_{m}(t),
$$

where $p_{m}=P_{m} u$ and $q_{m}=Q_{m} u, p_{m}$ and $q_{m}$ are large components and small components. Eq. (2.2a) is projected onto the space $P_{m} H$ and $Q_{m} H$, respectively, obtaining the following two equations,

$$
\frac{d p_{m}}{d t}+A p_{m}+P_{m} R\left(p_{m}+q_{m}\right)=P_{m} f, \quad p_{m}(0)=p_{m}^{0}=P_{m} u_{0},
$$




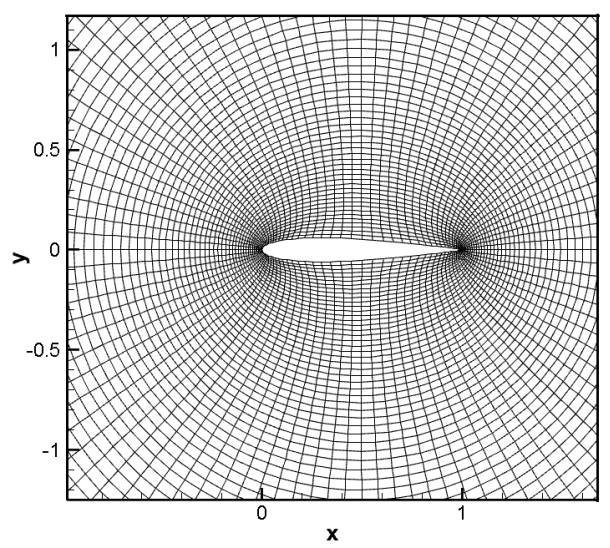

Figure 1: The mesh of the domain.

$$
\frac{d q_{m}}{d t}+A q_{m}+Q_{m} R\left(p_{m}+q_{m}\right)=Q_{m} f, \quad q_{m}(0)=q_{m}^{0}=Q_{m} u_{0}
$$

where $m$ is large enough, the time derivative term $\frac{d q_{m}}{d t} \approx 0$, and

$$
Q_{m} R\left(p_{m}+q_{m}\right) \approx Q_{m} R\left(p_{m}\right) .
$$

Finally, Eq. (2.5b) becomes

$$
A q_{m}+Q_{m} R\left(p_{m}\right)=Q_{m} f
$$

Thus obtaining

$$
q_{m}=\Phi\left(p_{m}\right)=A^{-1}\left[Q_{m} f-Q_{m} R\left(p_{m}\right)\right], \quad p_{m} \in P_{m} H,
$$

Eq. (2.7) results in $H$ the finite dimensional manifold.

\subsection{Multilevel finite element method and its basis}

Numerically, Inertial Manifold is implemented by using finite element method in such a way that spannings of both subspaces are given as coarse grid element space and incremental fine grid element space, which are finite. Meshing of the domain is carried out in terms of quadrangle elements, especially for the coarse grid finite element space given by Fig. 1. The initial element is represented as quadrangle elements having four nodes as initial mesh shown in Fig. 2, whereas the incremental refined mesh by addition of further 27 nodes in the quadrangle elements is shown in Fig. 3. Then, the fundamental finite element space is spanned by construction of hierarchical shape functions as basis functions in the initial element space accordingly.

The quadrangle elements with four nodes have the following shape functions,

$$
N_{1}=\frac{1}{4}(1-\xi)(1-\eta), \quad N_{2}=\frac{1}{4}(1+\xi)(1-\eta),
$$




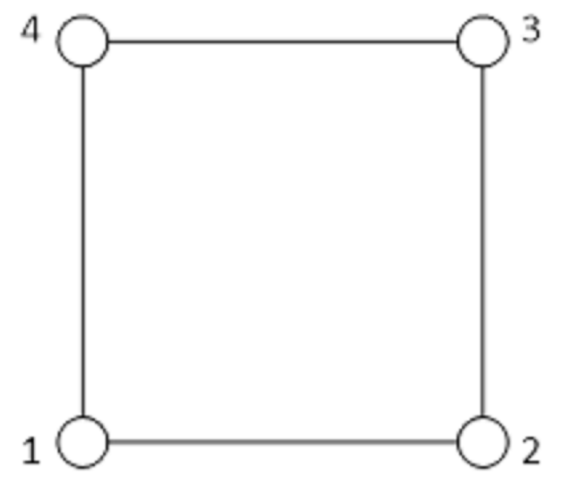

Figure 2: Quadrangle element with 4-nodes.

$$
N_{3}=\frac{1}{4}(1+\xi)(1+\eta), \quad N_{4}=\frac{1}{4}(1-\xi)(1+\eta) .
$$

The hierarchical shape function for 27 nodes can be expressed as following with the shape functions of first four nodes remaining the same,

$$
\begin{aligned}
& H_{1}=N_{1}, \quad H_{2}=N_{2}, \quad H_{3}=N_{3}, \quad H_{4}=N_{4}, \\
& \bar{H}_{5}=\frac{9}{120}\left(\xi^{2}-1\right)(3 \xi-2)\left(\eta^{2}-\eta\right)\left(4 \eta^{2}-1\right), \\
& \bar{H}_{6}=\frac{-9}{120}\left(\xi^{2}-1\right)(3 \xi+2)\left(\eta^{2}-\eta\right)\left(4 \eta^{2}-1\right), \\
& \bar{H}_{7}=\frac{-1}{60}\left(\xi^{2}+\xi\right)\left(9 \xi^{2}-1\right)\left(9 \xi^{2}-4\right)\left(\eta^{3}-\eta\right)(2 \eta-1), \\
& \bar{H}_{8}=\frac{1}{10}(\xi+1)\left(9 \xi^{2}-4\right)\left(\eta^{2}-1\right)\left(4 \eta^{2}-1\right), \\
& \bar{H}_{9}=\frac{-1}{60}\left(\xi^{2}+\xi\right)\left(9 \xi^{2}-1\right)\left(9 \xi^{2}-4\right)\left(\eta^{3}-\eta\right)(2 \eta+1), \\
& \bar{H}_{10}=\frac{-9}{120}(3 \xi+2)\left(\xi^{2}-1\right)\left(\eta^{2}+\eta\right)\left(4 \eta^{2}-1\right), \\
& \bar{H}_{11}=\frac{9}{120}\left(\xi^{2}-\xi\right)\left(3 \xi^{2}-2\right)\left(\eta^{2}+\eta\right)\left(4 \eta^{2}-1\right), \\
& \bar{H}_{12}=\frac{-1}{60}\left(\xi^{2}-\xi\right)\left(9 \xi^{2}-1\right)\left(9 \xi^{2}-4\right)\left(\eta^{3}-\eta\right)(2 \eta+1), \\
& \bar{H}_{13}=\frac{-1}{10}(\xi-1)\left(9 \xi^{2}-4\right)\left(\eta^{2}-1\right)\left(4 \eta^{2}-1\right), \\
& \bar{H}_{14}=\frac{-1}{60}\left(\xi^{2}-\xi\right)\left(9 \xi^{2}-1\right)\left(9 \xi^{2}-4\right)\left(\eta^{3}-\eta\right)(2 \eta-1), \\
& \bar{H}_{15}=\frac{9}{10}\left(\xi^{2}-\xi\right)\left(3 \xi^{2}-2\right)\left(9 \xi^{2}-1\right)\left(\eta^{3}-\eta\right)(2 \eta-1), \\
& \bar{H}_{16}=\frac{9}{32}\left(\xi^{3}-\xi\right)(3 \xi-1)\left(9 \xi^{2}-4\right)(2 \eta-1), \\
& \bar{H}_{17}=\frac{1}{8}\left(\xi^{2}-\xi\right)\left(9 \xi^{2}-1\right)\left(9 \xi^{2}-4\right)(2 \eta-1),
\end{aligned}
$$




$$
\begin{aligned}
& \bar{H}_{18}=\frac{9}{32}\left(\xi^{3}-\xi\right)(3 \xi+1)\left(9 \xi^{2}-4\right)(2 \eta-1), \\
& \bar{H}_{19}=\frac{9}{10}\left(\xi^{2}-\xi\right)(3 \xi+2)\left(9 \xi^{2}-1\right)\left(\eta^{3}-\eta\right)(2 \eta-1), \\
& \bar{H}_{20}=\frac{-9}{20}\left(\xi^{2}-1\right)(3 \xi+2)\left(\eta^{2}-\eta\right)\left(4 \eta^{2}-1\right), \\
& \bar{H}_{21}=\frac{3}{10}\left(\xi^{2}-\xi\right)(3 \xi+2)\left(9 \xi^{2}-1\right)\left(\eta^{3}-\eta\right)(2 \eta+1), \\
& \bar{H}_{22}=\frac{9}{32}\left(\xi^{3}-\xi\right)(3 \xi+1)\left(9 \xi^{2}-4\right)(2 \eta+1), \\
& \bar{H}_{23}=\frac{-1}{8}\left(\xi^{2}-1\right)\left(9 \xi^{2}-4\right)\left(9 \xi^{2}-1\right)(2 \eta+1), \\
& \bar{H}_{24}=\frac{9}{32}\left(\xi^{3}-\xi\right)(3 \xi-1)\left(9 \xi^{2}-4\right)(2 \eta+1), \\
& \bar{H}_{25}=\frac{3}{10}\left(\xi^{2}-\xi\right)(3 \xi-2)\left(9 \xi^{2}-1\right)\left(\eta^{3}-\eta\right)(2 \eta+1), \\
& \bar{H}_{26}=\frac{-9}{20}\left(\xi^{2}-\xi\right)(3 \xi-2)\left(\eta^{2}-1\right)\left(4 \eta^{2}-1\right), \\
& \bar{H}_{27}=\frac{-1}{4}\left(\xi^{2}-1\right)\left(9 \xi^{2}-4\right)\left(4 \eta^{2}-1\right) .
\end{aligned}
$$

In terms of Inertial Manifolds, the elements of velocity $u^{(e)}$ are decomposed into two components $y^{(e)}$ and $z^{(e)}$, which are large component and small component, respectively, reads,

$$
u^{(e)}=y^{(e)}+z^{(e)} .
$$

In summation notation, the above expression can be written as

$$
u^{(e)}=\sum_{1}^{4} H_{i} y_{i}^{(e)}, \quad z^{(e)}=\sum_{5}^{27} \bar{H}_{i} z_{i}^{(e)} .
$$

\section{Numerical scheme for Navier-Stokes equations}

The governing equations for the incompressible flow are

$$
\begin{aligned}
& \frac{\partial u_{j}}{\partial x_{j}}=0, \\
& \frac{\partial u_{i}}{\partial t}+u_{j} \frac{\partial u_{i}}{\partial x_{j}}=-\frac{1}{\rho} \frac{\partial p}{\partial x_{i}}+v \frac{\partial^{2} u_{i}}{\partial x_{j} \partial x_{j}} .
\end{aligned}
$$

Euler method is used to approach the time derivative as pressure-correction technique is implemented numerically for the governing equations, where $i=1,2 ; n$ is the step time

$$
\frac{\partial \hat{u}_{j}^{(n+1)}}{\partial x_{j}}=0
$$




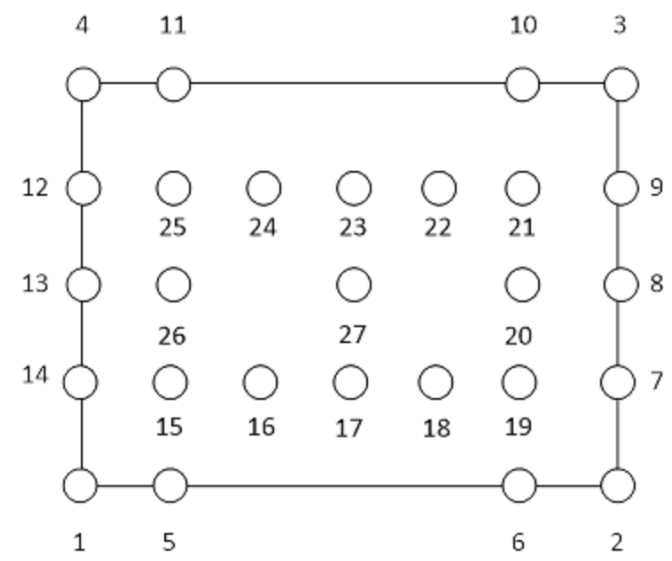

Figure 3: Quadrangle element with 27-nodes.

$$
\frac{\hat{u}_{i}^{(n+1)}-u_{i}^{(n)}}{\Delta t}+u_{j}^{(n)} \frac{\partial u_{j}^{(n)}}{\partial x_{j}}=-\frac{1}{\rho} \frac{\partial p^{(n+1)}}{\partial x_{i}}+v \frac{\partial^{2} u_{i}^{(n)}}{\partial x_{j} \partial x_{j}} .
$$

An intermediate velocity $\hat{u}$ is introduced in the implicit method, and then the momentum equation can be rewritten as

$$
\frac{\hat{u}_{i}-u_{i}^{(n)}}{\Delta t}+u_{j}^{(n)} \frac{\partial u_{j}^{(n)}}{\partial x_{j}}=-\frac{1}{\rho} \frac{\partial p^{(n)}}{\partial x_{i}}+v \frac{\partial^{2} u_{i}^{(n)}}{\partial x_{j} \partial x_{j}} .
$$

Subtracting Eq. (3.2b) from Eq. (3.3) as the quantity $p$ is explicit in the method, yields

$$
u_{i}^{n+1}-u_{i}=-\frac{\Delta t}{\rho}\left(\frac{\partial p^{(n+1)}}{\partial x_{i}}-\frac{\partial p^{(n)}}{\partial x_{i}}\right) .
$$

Introducing

$$
\begin{aligned}
& u_{i}^{n+1}-u_{i}=-\frac{\partial \phi}{\partial x_{i}} \\
& -\frac{\partial \phi}{\partial x_{i}}=-\frac{\Delta t}{\rho}\left(\frac{\partial p^{(n+1)}}{\partial x_{i}}-\frac{\partial p^{(n)}}{\partial x_{i}}\right) \\
& \frac{\partial \phi}{\partial x_{i}}=\frac{\Delta t}{\rho} \frac{\partial}{\partial x_{i}}\left(p^{(n+1)}-p^{(n)}\right), \\
& \frac{\rho}{\Delta t} \phi=\left(p^{(n+1)}-p^{(n)}\right) .
\end{aligned}
$$

Finally, pressure can be obtained as

$$
p^{(n+1)}=\frac{\rho}{\Delta t} \phi+p^{(n)}
$$


Also, in order to obtain $\phi$, we take the divergence of Eq. (3.5a) and applying continuity equation,

$$
\begin{aligned}
& \nabla\left(u_{i}^{n+1}-u_{i}\right)=\nabla\left(-\frac{\partial \phi}{\partial x_{i}}\right), \\
& \frac{\partial \hat{u_{1}}}{\partial x_{1}}+\frac{\partial \hat{u_{2}}}{\partial x_{2}}=\frac{\partial}{\partial x_{1}} \frac{\partial \phi}{\partial x_{1}}+\frac{\partial}{\partial x_{2}} \frac{\partial \phi}{\partial x_{2}}, \\
& \frac{\partial \hat{u_{1}}}{\partial x_{1}}+\frac{\partial \hat{u_{2}}}{\partial x_{2}}=\frac{\partial^{2} \phi}{\partial x_{1}}+\frac{\partial^{2} \phi}{\partial x_{2}},
\end{aligned}
$$

which is relevant to the poisson equation. So when substituting the value of $\phi$ into Eqs. (3.6) and (3.5d), both $u$ and $p$ can be obtained finally.

\section{Weak form of Navier-Stokes equation}

Before applying Multilevel Finite Element method to the governing equations, the weak form of governing equations should be obtained, i.e.,

$$
\begin{aligned}
& \int_{\Omega} \frac{\partial u_{j}}{\partial x_{j}} \delta p d \Omega=0 \\
& \int_{\Omega}\left[\frac{\partial u_{i}}{\partial t}+u_{j} \frac{\partial u_{i}}{\partial x_{j}}+\frac{1}{\rho} \frac{\partial p}{\partial x_{i}}-v \frac{\partial^{2} u_{i}}{\partial x_{j} \partial x_{j}}\right] \delta u_{i} d \Omega=0 .
\end{aligned}
$$

Using Greens Formulas both Eq. (4.1a) and Eq. (4.1b) can be rewritten as

$$
\begin{aligned}
& \int_{\Omega} u_{j} \frac{\partial}{\partial x_{j}}(\delta p) d \Omega=\int_{\Gamma_{1}} u_{n} \delta p d \Gamma \\
& \int_{\Omega}\left\{\left(\frac{\partial u_{i}}{\partial t}+u_{j} \frac{\partial u_{i}}{\partial x_{j}}\right) \delta u_{i}+\left[\frac{-p}{\rho} \delta_{i j}\right.\right. \\
& \left.\left.\quad+v\left(\frac{\partial u_{i}}{\partial x_{j}}+\frac{\partial u_{j}}{\partial x_{i}}\right)\right] \frac{\partial}{\partial x_{j}}\left(\delta u_{i}\right)\right\} d \Omega=\int_{\Gamma_{2}} \bar{p}_{i} \delta u_{i} d \Gamma .
\end{aligned}
$$

We first solve Eq. (4.4) for $i=1,2$

$$
\begin{gathered}
\int_{\Omega}\left\{\left(\frac{\partial u_{1}}{\partial t}+u_{1} \frac{\partial u_{1}}{\partial x_{1}}+u_{2} \frac{\partial u_{1}}{\partial x_{2}}\right) \delta u_{i}+\left[-\frac{p}{\rho} \frac{\partial}{\partial x_{1}}\left(\delta u_{i}\right)+v\left(2 \frac{\partial u_{1}}{\partial x_{1}} \frac{\partial}{\partial x_{1}}\left(\delta u_{i}\right)\right.\right.\right. \\
\left.\left.\left.+\frac{\partial u_{1}}{\partial x_{2}} \frac{\partial}{\partial x_{2}}\left(\delta u_{i}\right)+\frac{\partial u_{2}}{\partial x_{1}} \frac{\partial}{\partial x_{2}}\left(\delta u_{i}\right)\right)\right]\right\} d \Omega=\int_{\Gamma_{2}} \overline{p_{i}} \delta u_{i} d \Gamma, \\
\int_{\Omega}\left\{\left(\frac{\partial u_{2}}{\partial t}+u_{1} \frac{\partial u_{2}}{\partial x_{1}}+u_{2} \frac{\partial u_{2}}{\partial x_{2}}\right) \delta u_{i}+\left[-\frac{p}{\rho} \frac{\partial}{\partial x_{2}}\left(\delta u_{i}\right)+v\left(2 \frac{\partial u_{2}}{\partial x_{2}} \frac{\partial}{\partial x_{2}}\left(\delta u_{i}\right)\right.\right.\right. \\
\left.\left.\left.+\frac{\partial u_{2}}{\partial x_{1}} \frac{\partial}{\partial x_{1}}\left(\delta u_{i}\right)+\frac{\partial u_{1}}{\partial x_{2}} \frac{\partial}{\partial x_{1}}\left(\delta u_{i}\right)\right)\right]\right\} d \Omega=\int_{\Gamma_{2}} \overline{p_{i}} \delta u_{i} d \Gamma .
\end{gathered}
$$


By Inertial Manifold, the elements of velocity and pressure are decomposed mainly into two components, and applying the hierarchical basis functions, the above equations can be written as,

$$
\begin{aligned}
& \int_{\Omega} \frac{\partial \bar{u}_{1}}{\partial t} \bar{H}_{i} \bar{H}_{j} d \Omega+\int_{\Omega} u_{1} \frac{\partial u_{1}}{\partial x_{1}} \bar{H}_{i} d \Omega+\left(\int_{\Omega} u_{1} \bar{H}_{i} \frac{\partial \bar{H}_{j}}{\partial x_{1}} d \Omega\right) \bar{u}_{1}+\left(\int_{\Omega} \frac{\partial u_{1}}{\partial x_{1}} \bar{H}_{i} \bar{H}_{j} d \Omega\right) \bar{u}_{1} \\
& +\left(\int_{\Omega} \bar{H}_{i} \frac{\partial \bar{H}_{j}}{\partial x_{1}} \bar{H}_{l} d \Omega\right) \bar{u}_{1} \bar{u}_{1}+\int_{\Omega} u_{2} \frac{\partial u_{1}}{\partial x_{2}} \bar{H}_{i} d \Omega+\left(\int_{\Omega} u_{2} \bar{H}_{i} \frac{\partial \bar{H}_{j}}{\partial x_{2}} d \Omega\right) \bar{u}_{1} \\
& +\left(\int_{\Omega} \frac{\partial u_{1}}{\partial x_{2}} \bar{H}_{i} \bar{H}_{j} d \Omega\right) \bar{u}_{2}+\left(\int_{\Omega} \bar{H}_{i} \frac{\partial \bar{H}_{j}}{\partial x_{2}} \bar{H}_{l} d \Omega\right) \bar{u}_{2} \bar{u}_{1}-\frac{1}{\rho} \int_{\Omega} p \frac{\partial \bar{H}_{i}}{\partial x_{1}} d \Omega \\
& +\int_{\Omega} v\left[2 \frac{\partial u_{1}}{\partial x_{1}} \frac{\partial \bar{H}_{i}}{\partial x_{1}}+\frac{\partial u_{1}}{\partial x_{2}} \frac{\partial \bar{H}_{i}}{\partial x_{2}}+\frac{\partial u_{1}}{\partial x_{1}} \frac{\partial \bar{H}_{i}}{\partial x_{2}}\right] d \Omega+\left(\int _ { \Omega } v \left[2 \frac{\partial \bar{H}_{i}}{\partial x_{1}} \frac{\partial \bar{H}_{j}}{\partial x_{1}}\right.\right. \\
& \left.\left.+\frac{\partial \bar{H}_{i}}{\partial x_{2}} \frac{\partial \bar{H}_{j}}{\partial x_{2}}\right] d \Omega\right) \bar{u}_{1}+\left(\int_{\Omega} v \frac{\partial \bar{H}_{i}}{\partial x_{2}} \frac{\partial \bar{H}_{j}}{\partial x_{1}} d \Omega\right) \bar{u}_{2}=\int_{\Gamma_{2}} \bar{p}_{1 m} \psi_{m} \bar{H}_{i} d \Gamma \\
& \int_{\Omega} \frac{\partial \bar{u}_{2}}{\partial t} \bar{H}_{i} \bar{H}_{j} d \Omega+\int_{\Omega} u_{1} \frac{\partial u_{2}}{\partial x_{1}} \bar{H}_{i} d \Omega+\left(\int_{\Omega} u_{1} \bar{H}_{i} \frac{\partial \bar{H}_{j}}{\partial x_{1}} d \Omega\right) \bar{u}_{2}+\left(\int_{\Omega} \frac{\partial u_{2}}{\partial x_{1}} \bar{H}_{i} \bar{H}_{j} d \Omega\right) \bar{u}_{1} \\
& +\left(\int_{\Omega} \bar{H}_{i} \frac{\partial \bar{H}_{j}}{\partial x_{1}} \bar{H}_{l} d \Omega\right) \bar{u}_{1} \bar{u}_{2}+\int_{\Omega} u_{2} \frac{\partial u_{2}}{\partial x_{2}} \bar{H}_{i} d \Omega+\left(\int_{\Omega} u_{2} \bar{H}_{i} \frac{\partial \bar{H}_{j}}{\partial x_{2}} d \Omega\right) \bar{u}_{2} \\
& +\left(\int_{\Omega} \frac{\partial u_{2}}{\partial x_{2}} \bar{H}_{i} \bar{H}_{j} d \Omega\right) \bar{u}_{2}+\left(\int_{\Omega} \bar{H}_{i} \frac{\partial \bar{H}_{j}}{\partial x_{2}} \bar{H}_{l} d \Omega\right) \bar{u}_{2} \bar{u}_{2}-\frac{1}{\rho} \int_{\Omega} p \frac{\partial \bar{H}_{i}}{\partial x_{2}} d \Omega \\
& +\int_{\Omega} v\left[2 \frac{\partial u_{2}}{\partial x_{2}} \frac{\partial \bar{H}_{i}}{\partial x_{2}}+\frac{\partial u_{2}}{\partial x_{1}} \frac{\partial \bar{H}_{i}}{\partial x_{1}}+\frac{\partial u_{1}}{\partial x_{2}} \frac{\partial \bar{H}_{i}}{\partial x_{1}}\right] d \Omega+\left(\int _ { \Omega } v \left[2 \frac{\partial \bar{H}_{i}}{\partial x_{2}} \frac{\partial \bar{H}_{j}}{\partial x_{2}}\right.\right. \\
& \left.\left.+\frac{\partial \bar{H}_{i}}{\partial x_{1}} \frac{\partial \bar{H}_{j}}{\partial x_{1}}\right] d \Omega\right) \bar{u}_{2}+\left(\int_{\Omega} v \frac{\partial \bar{H}_{i}}{\partial x_{1}} \frac{\partial \bar{H}_{j}}{\partial x_{2}} d \Omega\right) \bar{u}_{1}=\int_{\Gamma_{2}} \bar{p}_{2 m} \psi_{m} \bar{H}_{i} d \Gamma .
\end{aligned}
$$

From Eq. (4.4a) and Eq. (4.4b), re-writing the terms as:

$$
\begin{aligned}
& \mathbf{A}=\int_{\Omega} \bar{H}_{i} \bar{H}_{j} d \Omega, \quad \mathbf{B}=\int_{\Omega} \bar{H}_{i} \frac{\partial \bar{H}_{j}}{\partial x_{1}} \bar{H}_{l} d \Omega, \quad \mathbf{C}=\int_{\Omega} \bar{H}_{i} \frac{\partial \bar{H}_{j}}{\partial x_{2}} \bar{H}_{l} d \Omega, \\
& \mathbf{F}_{1}=\int_{\Omega} u_{1} \bar{H}_{i} \frac{\partial \bar{H}_{j}}{\partial x_{1}} d \Omega+\int_{\Omega} \frac{\partial u_{1}}{\partial x_{1}} \bar{H}_{i} \bar{H}_{j} d \Omega+\int_{\Omega} u_{2} \bar{H}_{i} \frac{\partial \bar{H}_{j}}{\partial x_{2}} d \Omega+\int_{\Omega} v\left[2 \frac{\partial \bar{H}_{i}}{\partial x_{1}} \frac{\partial \bar{H}_{j}}{\partial x_{1}}+\frac{\partial \bar{H}_{i}}{\partial x_{2}} \frac{\partial \bar{H}_{j}}{\partial x_{2}}\right] d \Omega, \\
& \mathbf{F}_{2}=\int_{\Omega} \frac{\partial u_{1}}{\partial x_{2}} \bar{H}_{i} \bar{H}_{j} d \Omega+\int_{\Omega} v \frac{\partial \bar{H}_{i}}{\partial x_{1}} \frac{\partial \bar{H}_{j}}{\partial x_{2}} d \Omega, \quad \mathbf{F}_{3}=\int_{\Omega} \frac{\partial u_{2}}{\partial x_{1}} \bar{H}_{i} \bar{H}_{j} d \Omega+\int_{\Omega} v \frac{\partial \bar{H}_{i}}{\partial x_{2}} \frac{\partial \bar{H}_{j}}{\partial x_{1}} d \Omega, \\
& \mathbf{F}_{4}=\int_{\Omega} u_{1} \bar{H}_{i} \frac{\partial \bar{H}_{j}}{\partial x_{1}} d \Omega+\int_{\Omega} u_{2} \bar{H}_{i} \frac{\partial \bar{H}_{j}}{\partial x_{2}} d \Omega+\int_{\Omega} \frac{\partial u_{1}}{\partial x_{1}} \bar{H}_{i} b a r H_{j} d \Omega \\
& \quad+\int_{\Omega} v\left[2 \frac{\partial \bar{H}_{i}}{\partial x_{2}} \frac{\partial \bar{H}_{j}}{\partial x_{2}}+\frac{\partial \bar{H}_{i}}{\partial x_{1}} \frac{\partial \bar{H}_{j}}{\partial x_{1}}\right] d \Omega,
\end{aligned}
$$




$$
\begin{aligned}
\mathbf{E}_{1}=- & \int_{\Omega} u_{1} \frac{\partial u_{1}}{\partial x_{1}} \bar{H}_{i} d \Omega-\int_{\Omega} u_{2} \frac{\partial u_{1}}{\partial x_{2}} \bar{H}_{i} d \Omega+\frac{1}{\rho} \int_{\Omega} p \frac{\partial \bar{H}_{i}}{\partial x_{1}} d \Omega-\int_{\Omega} v\left[2 \frac{\partial u_{1}}{\partial x_{1}} \frac{\partial \bar{H}_{i}}{\partial x_{1}}+\frac{\partial u_{1}}{\partial x_{2}} \frac{\partial \bar{H}_{i}}{\partial x_{2}}\right. \\
& \left.+\frac{\partial u_{1}}{\partial x_{1}} \frac{\partial \bar{H}_{i}}{\partial x_{2}}\right] d \Omega+\int_{\Gamma_{2}} \bar{p}_{1 m} \psi_{m} \bar{H}_{i} d \Gamma \\
\mathbf{E}_{2}=- & \int_{\Omega} u_{1} \frac{\partial u_{2}}{\partial x_{1}} \bar{H}_{i} d \Omega-\int_{\Omega} u_{2} \frac{\partial u_{2}}{\partial x_{2}} \bar{H}_{i} d \Omega+\frac{1}{\rho} \int_{\Omega} p \frac{\partial \bar{H}_{i}}{\partial x_{2}} d \Omega-\int_{\Omega} v\left[2 \frac{\partial u_{2}}{\partial x_{2}} \frac{\partial \bar{H}_{i}}{\partial x_{2}}+\frac{\partial u_{2}}{\partial x_{1}} \frac{\partial \bar{H}_{i}}{\partial x_{1}}\right. \\
& \left.+\frac{\partial u_{1}}{\partial x_{2}} \frac{\partial \bar{H}_{i}}{\partial x_{1}}\right] d \Omega+\int_{\Gamma_{2}} \bar{p}_{2 m} \psi_{m} \bar{H}_{i} d \Gamma .
\end{aligned}
$$

Now solving Eq. (4.2a) for $i=1,2$

$$
\begin{aligned}
& \int_{\Omega} u_{1} \frac{\partial}{\partial x_{1}}(\delta p) d \Omega+\int_{\Omega} u_{2} \frac{\partial}{\partial x_{2}}(\delta p) d \Omega=\int_{\Gamma_{1}} u_{n} \delta p d \Gamma \\
& \int_{\Omega}\left[\left(u_{1 j}+\bar{u}_{1 j} \bar{H}_{j}\right) \frac{\partial}{\partial x_{1}} \psi_{k}+\left(u_{2 j}+\bar{u}_{2 j} \bar{H}_{j}\right) \frac{\partial}{\partial x_{2}} \psi_{k}\right] \bar{H}_{j} d \Omega=\int_{\Gamma_{1}} \bar{u}_{n i} H_{i} \psi_{k} d \Gamma \\
& \int_{\Omega} u_{1} \bar{H}_{i} \frac{\partial}{\partial x_{1}} \psi_{k} d \Omega+\left(\int_{\Omega} \bar{H}_{i} \bar{H}_{j} \frac{\partial}{\partial x_{1}} \psi_{k} d \Omega\right) \bar{u}_{1}+\int_{\Omega} u_{2} \bar{H}_{i} \frac{\partial}{\partial x_{2}} \psi_{k} d \Omega \\
& \quad+\left(\int_{\Omega} \bar{H}_{i} \bar{H}_{j} \frac{\partial}{\partial x_{1}} \psi_{k} d \Omega\right) \bar{u}_{2}=\int_{\Gamma_{1}} \bar{u}_{n i} H_{i} \psi_{k} d \Gamma .
\end{aligned}
$$

Re-writing the above equations as:

$$
\begin{aligned}
& \mathbf{M}_{1}=\int_{\Omega} \bar{H}_{i} \bar{H}_{j} \frac{\partial}{\partial x_{1}} \psi_{k} d \Omega, \quad \mathbf{M}_{2}=\int_{\Omega} \bar{H}_{i} \bar{H}_{j} \frac{\partial}{\partial x_{2}} \psi_{k} d \Omega, \\
& \mathbf{G}=\int_{\Gamma} \bar{u}_{n i} H_{i} \psi_{k} d \Gamma-\int_{\Omega} u_{1} \bar{H}_{i} \frac{\partial}{\partial x_{1}} \psi_{k} d \Omega-\int_{\Omega} u_{2} \bar{H}_{i} \frac{\partial}{\partial x_{2}} \psi_{k} d \Omega .
\end{aligned}
$$

Since the relationship between small and large components of the velocity in element can be expressed as

$$
u^{(e)}=y^{(e)}+z^{(e)}=\sum_{1}^{4} H_{i} y_{i}^{(e)}+\sum_{5}^{27} \bar{H}_{i} z_{i}^{(e)} .
$$

Also the pressure in the element is given as,

$$
P^{(e)}=\sum_{1}^{4} \psi_{i} P_{i}^{(e)}+\sum_{5}^{27} \bar{\psi}_{i} P_{i}^{(e)}
$$

We choose the weight function in Eq. (4.2b) as $\delta u_{i}=\bar{H}_{m}(m=5,6, \cdots, 27)$, and for Eq. (4.2a) we set $\delta p=\bar{\psi}_{n}(n=5,6, \cdots, 27)$. So Eqs. (4.2a) and (4.2b) can be rewritten as

$$
\mathbf{A} \dot{\bar{u}}_{1}+\mathbf{B} \bar{u}_{1} \bar{u}_{1}+\mathbf{C} \bar{u}_{2} \bar{u}_{1}+\mathbf{D}_{1} p+\mathbf{F}_{1} \bar{u}_{1}+\mathbf{F}_{2} \bar{u}_{2}=\mathbf{E}_{1}
$$




$$
\begin{aligned}
& \mathbf{A} \dot{\bar{u}}_{2}+\mathbf{B} \bar{u}_{1} \bar{u}_{2}+\mathbf{C} \bar{u}_{2} \bar{u}_{2}+\mathbf{D}_{2} p+\mathbf{F}_{3} \bar{u}_{1}+\mathbf{F}_{4} \bar{u}_{2}=\mathbf{E}_{2}, \\
& \mathbf{M}_{1} \bar{u}_{1}+\mathbf{M}_{2} \bar{u}_{2}=\mathbf{G} .
\end{aligned}
$$

The relationship between $y_{i}$ and $z_{i}$ is clearly expressed by Eqs. (4.5a) and (4.5b). We can get the values of $y_{i}$ since $z_{i}$ is known by the relationship. To approach the time derivative, Euler method is used,

$$
\dot{u}_{k}=\frac{u_{k}^{(i+1)}-u_{k}^{(i)}}{\Delta t}(k=1,2) .
$$

For expressing explicitly at $t=t_{i+1}$, we establish the following equations,

$$
\begin{gathered}
\mathbf{A} u_{1}^{(i+1)}+\Delta t\left(\mathbf{B} u_{1}^{(i+1)} u_{1}^{(i+1)}+\mathbf{C} u_{2}^{(i+1)} u_{1}^{(i+1)}+\mathbf{D}_{1} \bar{p}^{(i+1)}\right. \\
\left.+\mathbf{F}_{1} u_{1}^{(i+1)}+\mathbf{F}_{2} u_{2}^{(i+1)}\right)=\Delta t \mathbf{E}_{1}+\mathbf{A} u_{1}^{(i)} \\
\mathbf{A} u_{2}^{(i+1)}+\Delta t\left(\mathbf{B} u_{1}^{(i+1)} u_{2}^{(i+1)}+\mathbf{C} u_{2}^{(i+1)} u_{2}^{(i+1)}+\mathbf{D}_{2} \bar{p}^{(i+1)}\right. \\
\left.+\mathbf{F}_{3} u_{1}^{(i+1)}+\mathbf{F}_{4} u_{2}^{(i+1)}\right)=\Delta t \mathbf{E}_{2}+\mathbf{A} u_{2}^{(i)} .
\end{gathered}
$$

Newtons iterative formula is used for the $u^{(i+1)}$ step, since $u^{(i)}$ is known at the $i$ th step,

$$
J^{(i+1),(k)} v^{(i+1),(k+1)}=J^{(i+1),(k)} v^{(i+1),(k)}-R^{(i+1),(k)},
$$

where $J$ is Jacobean Matrix, $R=\left[\begin{array}{lll}R_{1} & R_{2} & R_{3}\end{array}\right]^{T}$ is the residual matrix and $v=\left[\begin{array}{lll}u_{1} & u_{2} & p\end{array}\right]^{T}$.

\subsection{Verification of numerical method}

The flow around NACA 0012 airfoil, which is a typical problem in computational fluid dynamics, is studied numerically. Verification of the above method will be analyzed by simulating flow over NACA 0012 airfoil at various angles with $R e=7 \times 10^{4}$. The angles of attack were ranged $\alpha=0^{\circ}-7^{\circ}$, obtaining lift coefficients. Fig. 4 shows a good agreement of the lift coefficients calculated with present method to the experimental data available in literature [31]. So we can conclude that the method used is suitable for studying the dynamics of flow over an airfoil. Fig. 5 presents the drag coefficients, computed at similar angles of attack as for lift coefficients, and compared with the experimental results [31]. It is observed that for laminar flow, skin friction drag is dominant as flow remains attached to the airfoil upto certain angles of attack showing less skin drag friction as compared to turbulent flow from experimental results shown in literature [31]. At high angle of attack, boundary layer separation starts when pressure drag becomes dominant and is considered less for turbulent flows in comparison with laminar flows. So, from Fig. 5, we can observe a sharp rise of curve at an angle of $6^{\circ}$ as separation starts for laminar flow. In many lift generating methods, the important quantity is the ratio of lift to drag presented in terms of $\mathrm{Cl} / \mathrm{Cd}$ verses angle of attack. Fig. 6 shows the most efficient angle of attack at $5^{\circ}$ for attaining maximum efficiency of NACA 0012 airfoil. After that the lift to drag ratio decreases rapidly as we reach separation. 


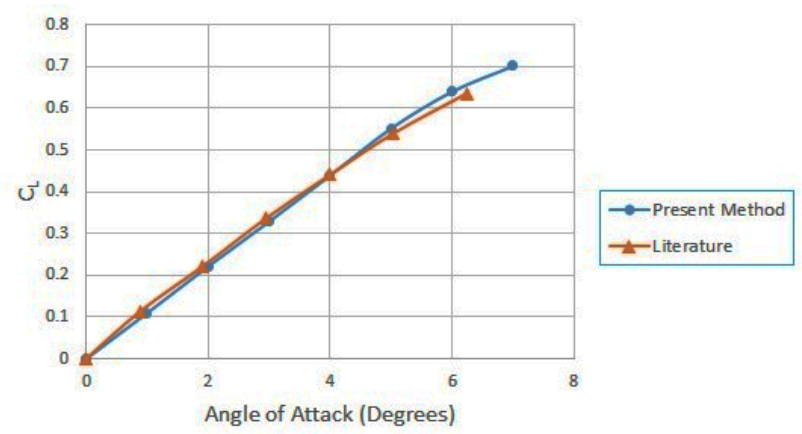

Figure 4: Comparisons between experiments and numerical results for Lift Coefficients.

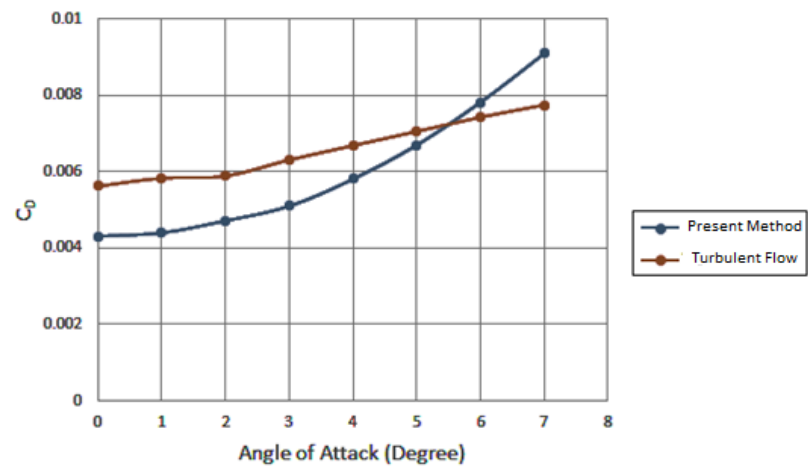

Figure 5: Comparisons between turbulent model and numerical results for Drag Coefficients.

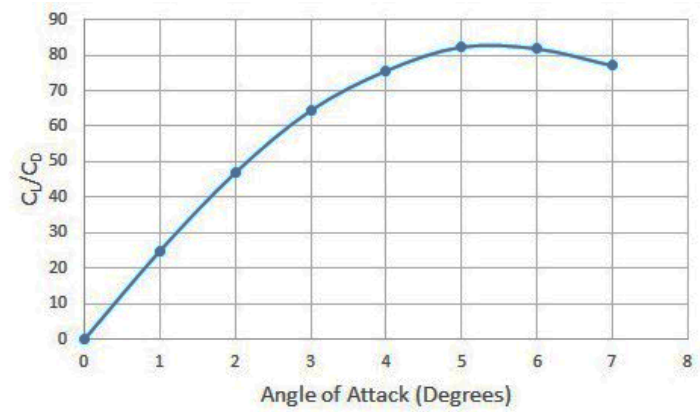

Figure 6: Ratio of $\mathrm{Cl} / \mathrm{Cd}$ for various angles of attack.

\section{Numerical results and discussion}

For numerical example, we have analyzed the flow around NACA 0012 airfoil with various angles of attack at $R e=7 \times 10^{4}$. The contours of pressure distribution and pressure coefficients using present numerical method have been discussed showing good results. At higher angles of attack, the flow is stimulated and studied numerically using commercial software ANSYS(Fluent) and present numerical method, and comparisons are given in 


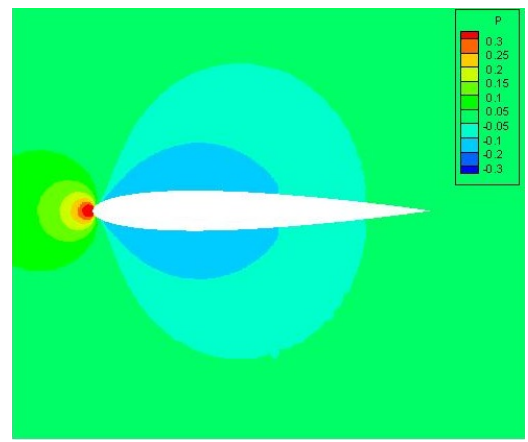

Figure 7: Pressure Distribution Contour at $0^{\circ}$.

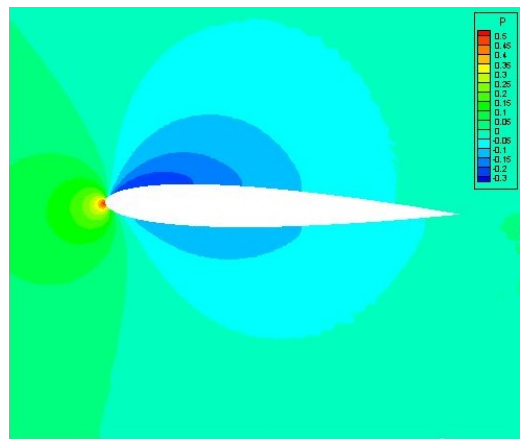

Figure 8: Pressure Distribution Contour at $2^{\circ}$.

order to show the efficiency of the scheme, especially the saving of computer time.

\subsection{Pressure distribution contours of NACA 0012 airfoil}

The distribution of pressure magnitude obtained by various angles of attack from simulations results by the present method are shown in the following Figs. 7-10. We can see there is a region of high pressure at the stagnation point (leading edge) since the airfoil is symmetrical. As the flow accelerates with increase in angle of attack we observe the pressure on the upper surface is less and the stagnation point changes its position where the pressure increases thus creating lift since velocity on the upper surface increases rapidly. Similar effects can be observed as the pressure profile increase on the lower surface of the airfoil upon increasing angles of attack until stall occurs. The distribution of flow created on the upper surface show the boundary layer separation with a smooth transition of flow on the upper surface of the airfoil.

\subsection{Distributions of pressure coefficients of NACA 0012 airfoil}

The distribution of pressure coefficient of NACA 0012 airfoil under different angles of attack is shown in the following Figs. 11-14. When $\alpha=0^{\circ}$, the pressure coefficient at the 


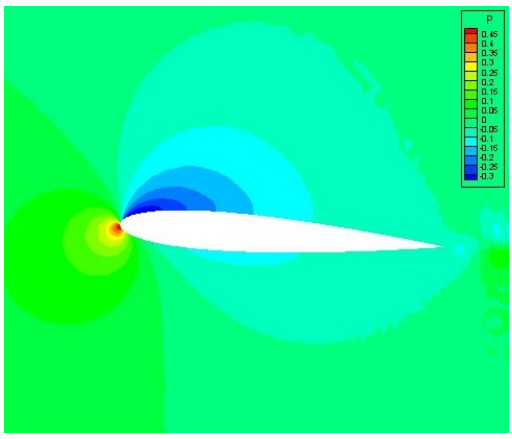

Figure 9: Pressure Distribution Contour at $4^{\circ}$.

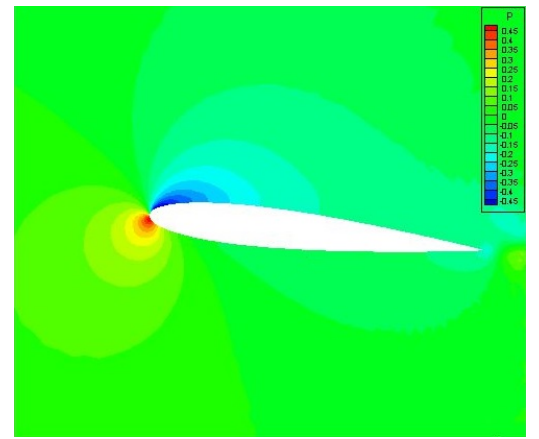

Figure 10: Pressure Distribution Contour at $6^{\circ}$.

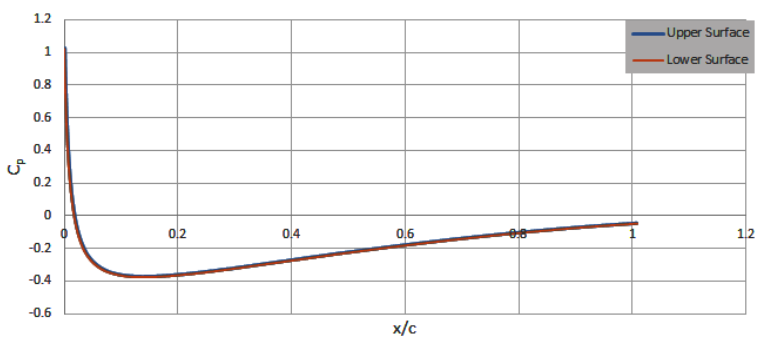

Figure 11: Pressure coefficient at $0^{\circ}$ angle of attack.

leading edge of the airfoil is maximum giving symmetrical variation of distribution with net zero velocity. When $\alpha=2^{\circ}$, the pressure coefficient on the lower surface increases as compared with the upper surface. When $\alpha=4^{\circ}$ pressure coefficient increase rapidly on the lower surface and low pressure on the upper surface contribute to increase in lift. When $\alpha=6^{\circ}$, the pressure coefficient at the trailing edge increase subsequently while on the upper surface low pressure induces smooth boundary layer separation before stall occurs at high angles of attack. With increase in angle of attack, greater is the difference of pressure coefficient between the lower and upper surface. We can also see that the coefficient of pressure difference is much larger on the front edge as compared with pressure on the rear edge of airfoil. 


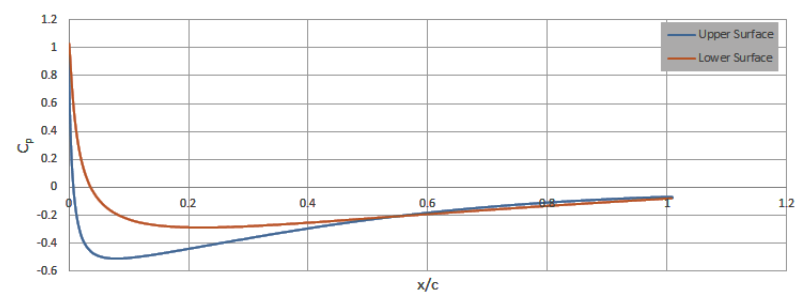

Figure 12: Pressure coefficient at $2^{\circ}$ angle of attack.

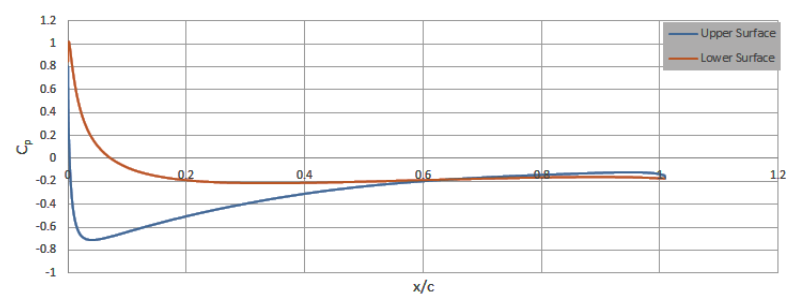

Figure 13: Pressure coefficient at $4^{\circ}$ angle of attack.

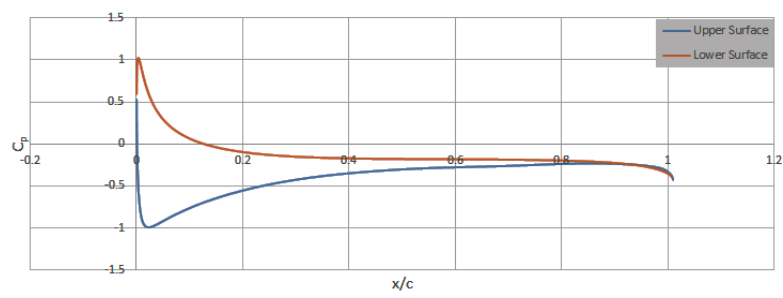

Figure 14: Pressure coefficient at $6^{\circ}$ angle of attack.

Table 1: Computational Coefficients of lift and drag at $10^{\circ}$ angle of attack-NACA0012.

\begin{tabular}{||l|cccc||}
\hline & $\begin{array}{c}\text { Lift Coefficient } \\
\left(C_{l}\right)\end{array}$ & $\begin{array}{c}\text { Drag Coefficient } \\
\left(C_{d}\right)\end{array}$ & $\begin{array}{c}\text { Max.Pressure } \\
\text { Coefficient }\end{array}$ & $\begin{array}{c}\text { CPU-time } \\
(\mathrm{sec})\end{array}$ \\
\hline ANSYS(Fluent) & 0.815 & 0.013 & 0.777 & 16280 \\
Present Method & 0.891 & 0.002 & 0.734 & 12816 \\
\hline
\end{tabular}

Table 2: Computational Coefficients of lift and drag at $12^{\circ}$ angle of attack-NACA0012.

\begin{tabular}{||l|cccc||}
\hline & $\begin{array}{c}\text { Lift Coefficient } \\
\left(C_{l}\right)\end{array}$ & $\begin{array}{c}\text { Drag Coefficient } \\
\left(C_{d}\right)\end{array}$ & $\begin{array}{c}\text { Max.Pressure } \\
\text { Coefficient }\end{array}$ & $\begin{array}{c}\text { CPU-time } \\
(\mathrm{sec})\end{array}$ \\
\hline ANSYS(Fluent) & 0.913 & 0.054 & 0.892 & 13656 \\
Present Method & 0.999 & 0.012 & 0.834 & 9594 \\
\hline
\end{tabular}

\subsection{Comparisons of computational time}

Computational time to be reduced, as for the initial step of attaining better simulations, the influence of mesh size is important and accurate numerical results are obtained as more 
nodes are added. Tables 1 and 2 shows the comparison of lift and drag coefficients with CPU-time obtained by the present method and ANSYS(Fluent)at higher angles of attack, i.e., $10^{\circ}$ and $12^{\circ}$, respectively. By the comparison, we conclude that the present method is more efficient in terms of computing cost, as we can see that the time taken by the present method is considerably less then ANSYS(Fluent).

\section{Conclusions}

Following Inertial Manifold, a dimension reduction method, based on multilevel finite element method with hierarchical basis, is presented for the Navier-Stokes Equation. The couplings between low modes and high modes have been considered and included by refining mesh domain using hierarchical finite element basis functions. The numerical simulation results indicate that the present method is feasible and efficient for the numerical analysis of complex flows with less computational time. The current work gives an efficient contribution towards model reduction method for the nonlinear dynamic systems with continuum mechanics, and reduces the original system to a system with less degreesof-freedom. As for further work, this method could be applied to flow with complicated domains.

Acknowledgments The research is supported by the National Basic Research Program of China (973 Program, Grant No. 2012CB026002) and the National Natural Science Foundation of China (Grant No. 51305355).

\section{References}

[1] R. Kамакоті and W. Shyy, Fluid-structure interaction for aeroelastic applications, Prog. Aerosp. Sci., 40(8) (2004), pp. 535-558.

[2] O. BENDiKSEN AND G. SEBER, Fluid-structure interactions with both structural and fluid nonlinearities, J. Sound Vib., 315(3) (2008), pp. 664-684.

[3] R. GORDNIER, High fidelity computational simulation of a membrane wing airfoil, J. Sound Vib., 25(5) (2009), pp. 897-917.

[4] P. Rojratsirikul, Z. WANG AND I. Gursul, Unsteady fluid-structure interactions of membrane airfoils at low Reynolds numbers, Exp. Fluids, 46(5) (2009), pp. 859-872.

[5] G. Rega And H. Troger, Dimension reduction of dynamical systems: methods, models, applications, Nonlinear Dyn., 41(1) (2005), pp. 1-15.

[6] A. Steindl and H. Troger, Methods for dimension reduction and their application in nonlinear dynamics, Int. J. Solids Struct., 38 (2001), pp. 2131-2147.

[7] J. Z. Zhang, Y. Liu AND D. M. Chen, Error estimate for influence of model reduction of nonlinear dissipative autonomous dynamical system on long-term behaviours, Appl. Math. Mech., 26 (2005), pp. 938-943.

[8] M. I. Friswell, J. E. T. Penny and S. D. Garvey, The application of the IRS and balanced realization methods to obtain reduced models of structures with local nonlinearities, J. Sound Vibr., 196 (1996), pp. 453-468.

[9] R. H. B. Fey, D. H. Von CAmpen And A. De Kraker, Long-term structural dynamics of mechanical system with local nonlinearities, ASME J. Vibr. Acoust., 118 (1996), pp. 147-163.

[10] M. KoRdT AND H. LusEBrink, Nonlinear order reduction of structural dynamic aircraft models, Aerospace Sci. Technol., 5 (2001), pp. 55-68. 
[11] P. M. A. Slaats, J. De Jongh and A. A. H. J. Sauren, Model reduction tools for nonlinear structural dynamics, Comput. Struct., 54 (1995), pp. 1155-1171.

[12] R. Temam, Infinite-Dimensional Dynamical System in Mechanics and Physics, Springer-Verlag, New York, 1997.

[13] E. S. Tiтi, On approximate inertial manifolds to the Navier-Stokes equations, J. Math. Anal. Appl., 149 (1990), pp. 540-557.

[14] F. Jauberteau, C. Rosier and R. Temam, A nonlinear Galerkin method for the Navier-Stokes equations, Comput. Meth. Appl. Mech. Eng., 80 (1990), pp. 245-260.

[15] I. D. ChuEshov, On a construction of approximate inertial manifolds for second order in time evolution equations, Nonlinear Anal. Theory Meth. Appl., 26 (1996), pp. 1007-1021.

[16] I. Mezic, Spectral Properties of Dynamical Systems, Model Reduction and Decomposition, Nonlinear Dynam., 41 (2005), pp. 309-325.

[17] A. V. Rezounenko, Inertial manifolds for retarded second order in time evolution equations, Nonlinear Anal., 51 (2002), pp. 1045-10054.

[18] J. Z. Zhang, R. Shen And M. Guanhua, Model Reduction on inertial manifolds for N-S equations approached by multilevel finite element method, Commun. Nonlinear Sci. Numer. Simul., 16 (2011), pp. 195-205.

[19] S. N. Chow AND K. Lu, Invariant manifolds for flows in Banach space, J. Diffe. Equ., 74 (2001), pp. 285-317.

[20] C. Foias, G. R. SEll And E. S. Titi, Exponential tracking and approximation of inertial manifolds for dissipative nonlinear equations, J. Dyn. Differ. Equ., 1 (1989), pp. 199-244.

[21] C. Foias, O. Manley and R. Temam, Modelling of the interaction of small and large eddies in two dimensional turbulent flows, Math. Mod. Numer. Anal., 22 (1988), pp. 93-118.

[22] G. Haller and S. Ponsioen, Exact model reduction by a slow-fast decomposition of nonlinear mechanical systems, Nonlinear Dyn., (2017), pp. 1-31.

[23] O. Schmidtmann, Modelling of the interaction of lower and higher modes in two-dimensional MHD-equations, Nonlinear Anal. Theory, Meth. Appl., 26 (1996), pp. 41-54.

[24] W. Kang, J. Z. Zhang, R. Shen And L. Penfei, Nonlinear Galerkin method for low-dimensional modeling of fluid dynamic system using POD modes, Commun. Nonlinear Sci. Numer. Simul., 22 (2015), pp. 943-952.

[25] C. R. Laing, A. McRobie And J. M. T. Thompson, The post-processed Galerkin method applied to non-linear shell vibrations, Dynam. Stabil. Syst., 14 (1999), pp. 163-181.

[26] A. V. Rezounenko, Inertial manifolds for retarded second order in time evolution equations, Nonlinear Anal., 51 (2002), pp. 1045-1054.

[27] J. Z. Zhang, Y. Liu, P. F. Lei AND X. Sun, Dynamic snap-through buckling analysis of shallow arches under impact load based on approximate inertial manifolds, Dyn. Continuous, Discrete Impuls. Syst. Series-B(DCDIS-B), 14 (2007), pp. 287-291.

[28] C. H. Whiting And K. E. JAnsen, A Stabilized finite element methods for incompressible NavierStokes equation using a hierarchical basis, Int. J. Numer. Meth. Fluids, 35 (2001), pp. 93-116.

[29] P. KRYSL, E. GRINSPUN AND P. SCHRODER, Natural hierarchical refinement for finite element methods, Int. J. Numer. Meth. Eng., 56 (2003), pp. 1109-1124.

[30] E. BAnk, F. Dupont And H. Yserentant, The hierarchical basis multigrid method, Numerische Mathematik Springer, 52 (1988), pp. 427-456.

[31] I. H. Аввотt And A. E. Von Doenhoff, Theory of Wing Sections, Dover Publishing, New York, 1959. 